\title{
Design of Panel Pattern Array in LED Flat Light for Improvement of Illuminance
}

\author{
Won-Sik Choi* and Si-Hyun Park ${ }^{\dagger}$
}

\begin{abstract}
A pattern array integrated on the front panel of a LED flat light was designed to improve the illuminance. Various pattern shapes were fabricated, including square pillar, hexagonal pillar, circular pillar, and hemispheres. Among them, the hemispherical pattern shape showed the highest improvement in the illuminance of the LED flat light due to a decrease in the total internal reflection of light. Using both a ray-tracing simulation and an experiment with a hardware fabrication, the optimal conditions in the pattern size of the hemispherical pattern array for the maximum improvement of the illuminance were investigated.
\end{abstract}

Keywords: LED flat light, Panel pattern, Illuminance, Ray-tracing analysis

\section{Introduction}

Lighting emitting diodes (LEDs) are used widely in many areas because of their many advantages, such as high energy conversion efficiency, high response rates, wide range of wavelengths (colors), and small size that secures a high degree of freedom in design. Accordingly, the LEDs can be applied to general lighting both indoors and outdoors, emotional lighting, automotive lightings, LCD backlights, visible light communication, diagnosis/therapy, healthcare, ultraviolet sterilization, Li-Fi (light-Fidelity), and plant factories. Among these applications, the LED lighting is particularly applicable and marketable, and a wide variety of LED lighting fixtures has been developed. In terms of the geometrical forms, LED flat lights are one of the forms that can make the best use of a range of advantages of LEDs, and they are applied to the products of high usability.

The light extraction efficiency of LEDs is of great importance. Basically the light extraction efficiency in the level of the LED chips is related to the difficulty of photons generated within the semiconductor materials to escape from the semiconductors due to the high refractive index. In actual InGaN-based LEDs with a simple regular hexahedron, the light extraction efficiency is as low as $4 \%$ of the total light generation [1]. Several studies have been conducted to improve the light extraction efficiency at the level of chips, such as chip shaping [2], patterned sapphire substrates [3-7], photonic crystals [8,9], surface roughening [10-13], and anti-/high-reflection surfaces [14-17]. In addition, the light extraction efficiency is an important factor at the level of lighting fixtures. In this area, the light extraction efficiency depends on the structure of the light

$\dagger$ Corresponding Author: Dept. of Electronic Engineering, Yeungnam University, Korea. (sihyun_park@ynu.ac.kr)

* Dept. of Electronic Engineering, Yeungnam University, Korea. (ws_choi@ynu.ac.kr)

Received: February 27, 2016; Accepted: April 14, 2016 fixtures, and in the case of LED flat lights, enhanced efficiency may be expected when certain patterns are designed to be integrated on the front panel $[18,19]$. On the other hand, there are still no reports on the optimization of patterns in relation to the improvement of the light extraction efficiency.

This study examined the optimal patterns of the front panels on the light extraction efficiency with the aim of improving the light extraction efficiency of LED flat lights.

\section{Experiments}

The basic structure of the LED flat light designed in this study is as follows; an aluminum plate was used on the sides and bottom of this cube housing, whose inner size is $300 \times 300 \times 52 \mathrm{~mm}^{3}$. For the inner surface of this housing, common white paint was used, which is common for light fixtures. For the panel in front of the light fixture, a polycarbonate plate, whose size was $300 \times 300 \times 2 \mathrm{~mm}^{3}$, was used. The light source of the LED was SFT825Z-S model PKG LED of Seoul Semiconductor Co., Ltd., which utilizes 3 RGB chips for the white color. A total of 100 LED light sources were arranged $10 \times 10$ at the same interval inside the housing. The actual patterns were fabricated on the polycarbonate panel using a 3D image processing device. The LED flat light illuminance was measured $1 \mathrm{~m}$ away from the structure using an illuminometer (Konica Minolta, Inc. T-10A).

A ray-tracing numerical simulation was conducted on the LED flat light structure using LightTools (Synopsys Co., Ltd.). The optical characteristics of the inner surface were those of common white paint; the reflectivity of the Lambertian surface was $92 \%$ and the absorption was $8 \%$. The Fresnel reflectance surface was applied to the front panel [20].

Fig. 1 shows a schematic diagram and photograph of 


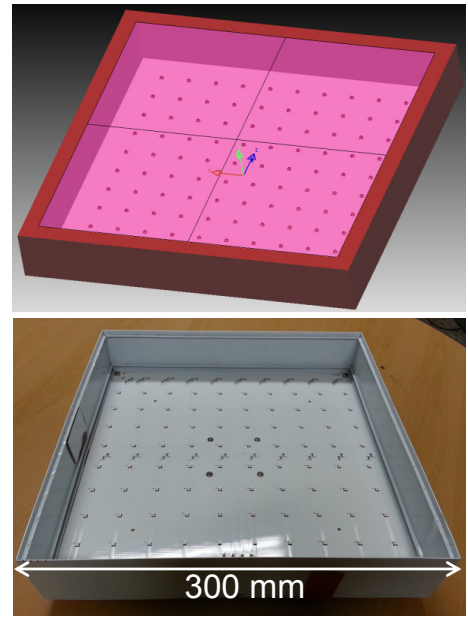

Fig. 1. Schematic diagram and photograph of the LED flat light
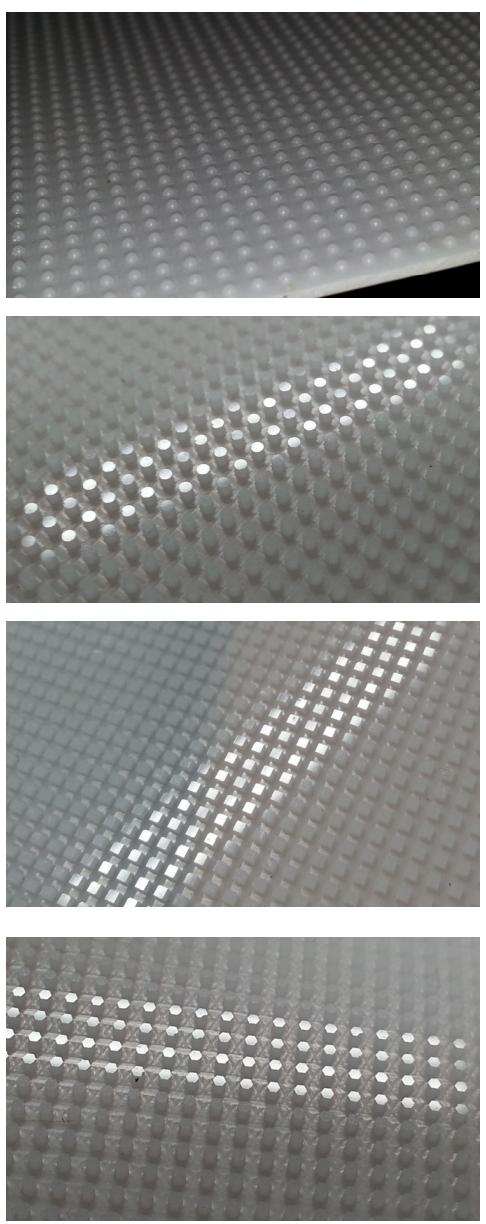

Fig. 2. Arrangement of the patterns on the front panel surface. Hemispherical shape, cylinder, square pillar, and hexagonal pillar patterns from the top to the bottom

the LED flat light. Fig. 2 presents photographs of the integrated patterns on the front panel surface. At the bottom side of the front panel, patterns of an extruded type were integrated. The height, width, and interval of each pattern were the same as $1 \mathrm{~mm}, 1 \mathrm{~mm}$, and $2 \mathrm{~mm}$, respectively (for hemisphere, the height is $0.5 \mathrm{~mm}$ )(See Fig. 5 for definitions of the height, width, and interval of pattern). Various types of patterns including the hemispherical shape, cylinder, square pillar, and hexagonal pillar were attempted to determine the optimal one in terms of the light extraction efficiency.

\section{Results and Discussion}

Fig. 3 shows the simulated illuminance (a) when the front panel of the LED flat light had patterns and (b) when it did not, respectively. When there were patterns, the illuminance was improved by up to $\sim 2.8$ times compared to that when there were no patterns. This is because the patterns integrated on the panel reduced the total internal reflection inside after the light from the LED light source came into the panel and the patterns also induced light scattering, which increased the final light extraction efficiency of the LED flat light. The pattern used in the simulation of Fig. 3(a) was hemispherical: base diameter of $1 \mathrm{~mm}$, hemispherical height of $0.7 \mathrm{~mm}$, and distance between patterns of $1 \mathrm{~mm}$.

As confirmed in Fig. 3, the front panel patterns improved the illuminance of the LED flat light. The illuminance of the LED flat lights when the hemispherical shape, square pillar, hexagonal pillar, and cylinder were applied was also investigated.

First the hardware of each pattern was fabricated as shown in Fig. 2 and the illuminance was measured. In

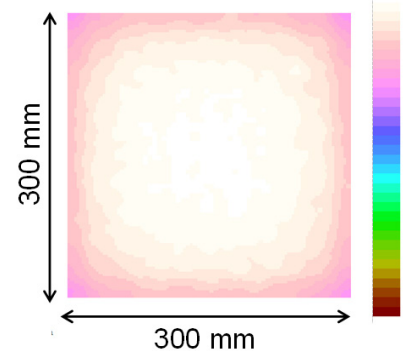

(a)

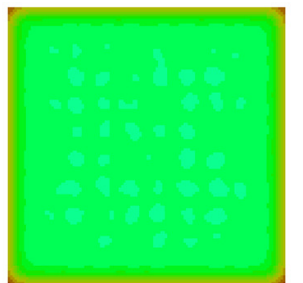

(b)

Fig. 3. Output illuminance values in the simulation: (a) when the front panel of the LED flat light has patterns (hemispherical patterns); and (b) when the front panel has no patterns 


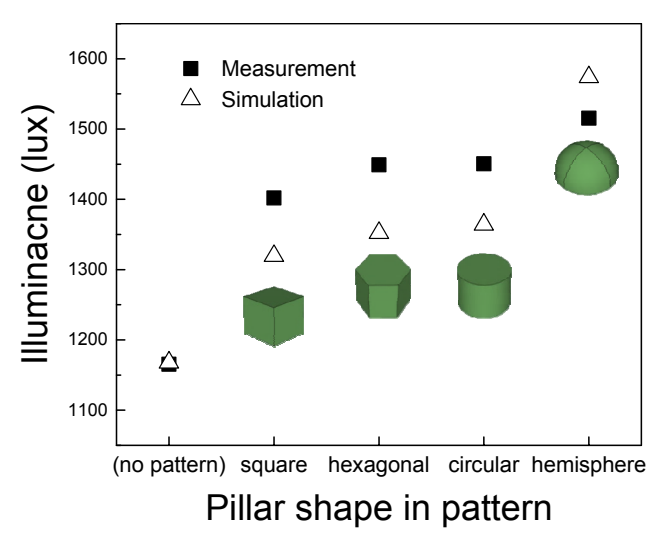

Fig. 4. Result of the measurements and simulation of illuminance on the front panel of the LED flat light with the hemispherical shape, square pillar, hexagonal pillar, and cylinder patterns, respectively.

addition, the illuminance simulation was conducted for each pattern using LightTools. Fig. 4 shows the results. For the four different patterns, the height, width, and interval were the same $(1 \mathrm{~mm}, 1 \mathrm{~mm}$, and $2 \mathrm{~mm}$, respectively). Therefore, the difference that only originated from the shape could be examined.

When the four patterns were used, the illuminance was basically improved compared to when no pattern was used. Among these, the hemispherical pattern showed the highest level of illuminance. The numerical simulation indicated that the hemispherical shape involved a less critical angle for the total internal reflection to the incident rays than the other patterns, which decreased the total internal reflection most efficiently and improved the light extraction efficiency.

As the light extraction efficiency of the hemispherical pattern was the highest, the optimal condition of its pattern size was examined. The variables included the interval, height, and width of the pattern. A numerical simulation was conducted to measure the change in illuminance depending on the variables. First of all, the pattern interval was adjusted to 1,2, and $3 \mathrm{~mm}$ after the height and width was fixed to $1 \mathrm{~mm}$ each. The number of hemispherical patterns decreased with increasing interval. As a result, the output illuminance decreased accordingly as shown in Fig. 5 (a). The pattern height increased gradually from $0.1 \mathrm{~mm}$ to $1.5 \mathrm{~mm}$ with the interval and width fixed to $1 \mathrm{~mm}$ each. As shown in (b), the maximum illuminance was observed at a certain hemispherical height. Initially, the level of illuminance increased with increasing hemispherical height, and the maximum illuminance was measured at the point of $\sim 0.7 \mathrm{~mm}$. The level then decreased with increasing hemispherical height. Finally, the width increased gradually from $0.1 \mathrm{~mm}$ to $1.5 \mathrm{~mm}$ with the interval and height fixed to $1 \mathrm{~mm}$ and $0.7 \mathrm{~mm}$, respectively. As shown in Fig. 5(c), the maximum illuminance was observed at a certain width, $1.0 \mathrm{~mm}$ in this case.

Regarding the size of the hemispherical pattern, the
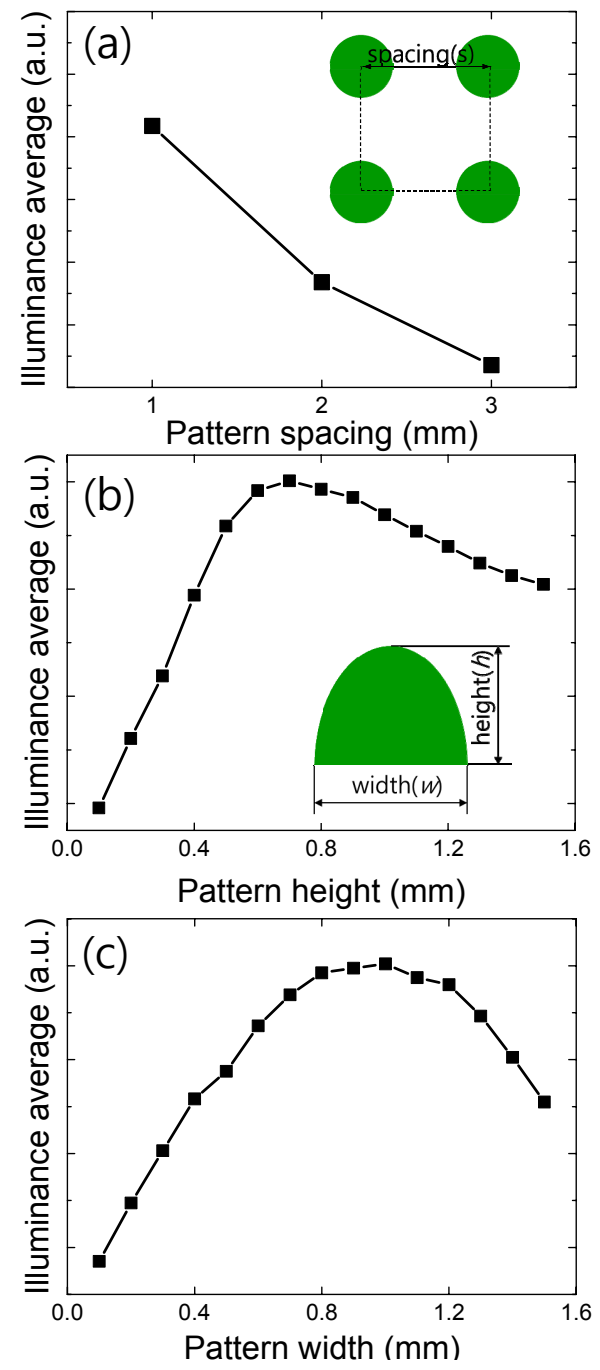

Fig. 5. (a) Changes in the LED flat light illuminance depending on the pattern interval; (b) Changes in the LED flat light illuminance depending on the pattern height; (c) Changes in the LED flat light illuminance depending on the pattern width.

maximum illuminance was measured when the pattern to pattern interval, width and height was fixed to $1 \mathrm{~mm}, 1 \mathrm{~mm}$, and $0.7 \mathrm{~mm}$, respectively. In addition, when conventional methods of panel arrangements were compared with our results, for example, 1-D stripe pattern with triangular cross section, it was obtained our hemispherical pattern still shows the highest light output performance with over 1.2 times.

\section{Conclusion}

To enhance the light extraction efficiency of the LED flat lights, an integrated set of patterns was applied to the front panel. Among the various patterns, the hemispherical form showed the highest level of illuminance, and the 
numerical simulation showed that the hemispherical shape effectively reduced the total internal reflection within the panel and increased the light extraction efficiency better than the other patterns. For the hemispherical shape that showed the optimal illuminance improvement, the pattern that would best optimize the light extraction efficiency was sought by adjusting the pattern size. As a result, the highest efficiency was achieved when the interval, width and height was $1 \mathrm{~mm}, 1 \mathrm{~mm}$, and $0.7 \mathrm{~mm}$, respectively. These findings are expected to be useful for panel pattern designs that will improve the light extraction efficiency of LED flat lights.

\section{Acknowledgements}

This work was supported by the 2015 Yeungnam University Research Grant.

\section{References}

[1] E. F. Schubert, Light-Emitting Diode, 2nd edn, University Press. pp. 91-93, 2006.

[2] Stuart E. Brinkley, Christopher Lalau Keraly, Junichi Sonoda, Claude Weisbuch, Jim S. Speck, Shuji Nakamura, and Steven P. DenBaars, "Chip Shaping for Light Extraction Enhancement of Bulk c-Plane Light-Emitting Diodes", Appl. Phys. Express, Vol. 5, No. 3, pp. 032104, Mar. 2012.

[3] Xiao-Hui Huang, Jian-Ping Liu, Jun-Jie Kong, Hui Yang and Huai-Bing Wang, "High-efficiency InGaNbased LEDs grown on patterned sapphire substrates", Opt. Express, Vol. 19, No. S4, pp. A949-A955, July 2011.

[4] Jae-Hoon Lee, J. T. Oh, Y. C. Kim, and Jung-Hee Lee, "Stress reduction and enhanced extraction efficiency of GaN-based LED grown on cone-shape patterned sapphire", IEEE Photonics Technol. Lett., Vol. 20, No. 18, pp. 1563-1565, Sep. 2008.

[5] Y. K. Su, J. J. Chen, C. L. Lin, S. M. Chen, W. L. Li, C. C. Kao, "Pattern-size dependence of characteristics of nitride-based LEDs grown on patterned sapphire substrates", J. Cryst. Growth, Vol. 311, No. 10, pp. 2973-2976, May 2009.

[6] Yu-Ting Hsu, Cheng-Chang Yu, Kai-Feng Huang, Wen-How Lan, Jing-En Huang, Jia-Ching Lin, and Wen-Jen Lin, "Improved Output Power of NitrideBased Light-Emitting Diodes With Convex-Patterned Sapphire Substrates", IEEE Photonics Technol. Lett., Vol. 24, No. 19, pp. 1686-1688, Oct. 2012.

[7] Xiao-Hang Li, Renbo Song, Yik-Khoon Ee, Pisist Kumnorkaew, James F. Gilchrist,and Nelson Tansu, "Light Extraction Efficiency and Radiation Patterns of III-Nitride Light-Emitting Diodes With Colloidal Microlens Arrays With Various Aspect Ratios", IEEE
Photonics J., Vol. 3, No. 3, pp.489-499, June 2011.

[8] J.-Y. Chen, J.-Y. Yeh, L.-W. Chen, Y.-G. Li, and C.-C. Wang, "Design and Modeling for Enhancement of Light Extraction in Light-Emitting Diodes with Archimedean Lattice Photonic Crystals", Prog. Electromagn. Res. B, Vol. 11, pp. 265-279, 2009.

[9] Dong-Ho Kim, Chi-O Cho, Yeong-Geun Roh, Heonsu Jeon, Yoon Soo Park, Jaehee Cho, Jin Seo Im, Cheolsoo Sone, Yongjo Park, Won Jun Choi, and QHan Park, "Enhanced light extraction from GaNbased light-emitting diodes with holographically generated two-dimensional photonic crystal patterns", Appl. Phys. Lett., Vol. 87, No. 20, pp. 203508, Nov. 2005.

[10] Chul Huh, Kug-Seung Lee, Eun-Jeong Kang, and Seong-Ju Park, "Improved light-output and electrical performance of InGaN-based light-emitting diode by microroughening of the p-GaN surface", J. Appl. Phys., Vol. 93, No. 11, pp. 9383-9385, June 2003.

[11] Wei Chih Peng and YewChung Sermon Wu, "Enhanced performance of an InGaN-GaN lightemitting diode by roughening the undoped-GaN surface and applying a mirror coating to the sapphire substrate", Appl. Phys. Lett., Vol. 88, No. 18, pp. 181117, May 2006.

[12] T. Fujii, Y. Gao, R. Sharma, E. L. Hu, S. P. DenBaars, and S. Nakamura, "Increase in the extraction efficiency of GaN-based light-emitting diodes via surface roughening", Appl. Phys. Lett., Vol. 84, No. 6, pp. 855-857, Feb. 2004.

[13] Tak Jeong, Hyung-Jo Park, Ki Chang Jung, Jong Hyeob Baek, Jun-Seok Ha, Won-Sik Choi, Si-Hyun Park, "Light output improvement of $10 \mathrm{~W}$ operated vertical LEDs via surface roughening using a commercialized developer", J Mater Sci: Mater Electron, Vol. 26, No. 6, pp. 3397-3402, June 2015.

[14] Jong Kyu Kim, Thomas Gessmann, Hong Luo, and E. Fred Schuberta, "GaInN light-emitting diodes with $\mathrm{RuO}_{2} / \mathrm{SiO}_{2} / \mathrm{Ag}$ omni-directional reflector", Appl. Phys. Lett., Vol. 84, No. 22, pp. 4508-4510, May 2004.

[15] J.-Q. Xi, Manas Ojha, J. L. Plawsky, W. N. Gill, Jong Kyu Kim and E. F. Schubert, "Internal high-reflectivity omni-directional reflectors", Appl. Phys. Lett., Vol. 87, No.3, pp. 031111, Jul. 2005.

[16] Jong Kyu Kim, Thomas Gessmann, E. Fred Schubert, J. -Q. Xi, Hong Luo, Jaehee Cho, Cheolsoo Sone, and Yongjo Park, "GaInN light-emitting diode with conductive omnidirectional reflector having a lowrefractive-index indium-tin oxide layer", Appl. Phys. Lett., Vol. 88, No. 1, pp. 013501, Jan. 2006.

[17] Jong Kyu Kim, J.-Q. Xi, Hong Luo, E. Fred Schubert, Jaehee Cho, Cheolsoo Sone, and Yongjo Park, "Enhanced light-extraction in GaInN near-ultraviolet light-emitting diode with Al-based omnidirectional reflector having NiZn/Ag microcontacts", Appl. Phys. Lett., Vol. 89, No. 14, pp. 141123, Oct. 2006. 
[18] Young Chul Kim, Tae-Sik Oh, Yong Min Lee, "Optimized pattern design of light-guide plate (LGP)", Opt. Appl., Vol. XLI, No. 4, pp. 863-872, Dec. 2011

[19] Young Hyun Ju, Ji-Hee Park, Jeong Ho Lee, Ji-Young Lee, Kie-Bong Nahm, Jae-Hyeon Ko and Joong Hyun Kim, "Study on the Simulation Model for the Optimization of Optical Structures of Edge-lit Backlight for LCD Applications", J. Opt. Soc. Korea, Vol. 12, No. 1, pp. 25-30, Mar. 2008.

[20] B. E. A. Saleh, M. C. Teich, Fundamentals of Photonics, 1st edn, Wiley. pp. 203-210, 1991.

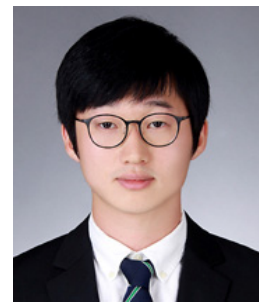

Won-Sik Choi He received B.S degree in photonic engineering from Chosun University, Korea in 2010. He is currently working toward the Ph.D. degree in the Yeungnam University, Korea. His research interests are fabrication, and characterization of LEDs.

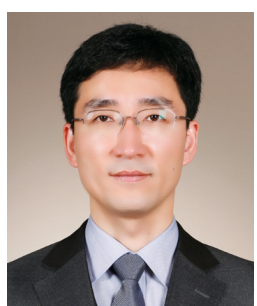

Si-Hyun Park He received Ph.D. degree specialized in optoelectronics from Seoul National University in 2004. $\mathrm{He}$ is currently working as Associate Professor in Department of Electronic Engineering, Yeungnam University, Korea. His current research interests are design, fabrication and characterization of optical active and passive devices such as LED and solar cell. 